\title{
Myocardial Injury Is Associated with Higher Morbidity and Mortality in Patients with 2019 Novel Coronavirus Disease (COVID-19)
}

\author{
Kamla AI-Wahaibi ${ }^{1}$ (D) Yahya Al-Wahshi ${ }^{2} \cdot$ Osman Mohamed Elfadil $^{2}$
}

Accepted: 1 October 2020 /Published online: 9 October 2020

(C) Springer Nature Switzerland AG 2020

\begin{abstract}
COVID-19 pandemic, a global health disaster, has resulted in substantial morbidity and mortality across the globe since emerging on December 2019. Studies have shown that cardiovascular manifestations and complications linked to COVID-19 can be attributed to unfavorable clinical outcome and poor prognosis. Adult patients with laboratory-confirmed COVID-19 requiring hospitalization in participating centers between March and June 2020 were included. Data including demographics, laboratory findings, comorbidities, treatments and interventions were collected. Mortality and clinical outcomes in patients with and without cardiac injury were compared. A total of 143 hospitalized patients with confirmed COVID-19 were included (86.7\% male; age $49.36 \pm 15.32$ years). Cardiovascular diseases (CVDs) including hypertension, cardiomyopathy, coronary heart disease, and rhythm disturbances were noted in $34.3 \%$ of the study population and $21.7 \%$ had cardiac injury. In comparison with patients without cardiac injury, patients with cardiac injury were older (59 [33-89] vs 47 [22-94] years; $P<0.0001$ ) and had more comorbidities and cardiovascular (CV) risk factors (hypertension in $61.3 \%$ vs $24.1 \% ; \mathrm{P}<0.0001$, chronic heart failure in $16.1 \%$ vs $0 \% ; P<0.00001$, diabetes mellitus $54.8 \%$ vs $31.3 \% ; P 0.015$, COPD/asthma $19.4 \%$ vs $3.6 \% ; P 0.002$ ); more patients with cardiac injury required invasive mechanical ventilation ( $77.4 \%$ vs $38.4 \% ; P$ 0.00012). Complications were more prevalent in patients with cardiac injury than those without cardiac injury and included acute respiratory distress syndrome (87\% vs $42.9 \%$; $P<0.00001)$, acute kidney injury $(67.7 \%$ vs $11.6 \% ; P<0.00001)$, and anemia $(38.7 \%$ vs $3.6 \% ; P<0.00001)$. The need for renal replacement therapy was also higher in patients with cardiac injury $(48.4 \%$ vs $3.6 \% ; P<0.00001)$. Noticeably, patients with cardiac injury had higher mortality than those without cardiac injury $(53.3 \%$ vs $7.1 \% ; P<0.00001)$. In summary, myocardial injury is common among hospitalized patients with COVID-19 in Oman in relation to older patients with more CV risk factors and comorbidities, and is associated with higher risk of in-hospital mortality and unfavorable clinical outcomes.
\end{abstract}

Keywords Cardiac injury $\cdot$ Myocardial injury $\cdot$ COVID-19 · Oman

\section{Introduction}

Coronavirus disease 2019 (COVID-19) is an infection of the respiratory tract caused by a newly emerging coronavirus, SARS-CoV-2, which was first identified in December 2019 at Wuhan, China. Based on genetic virus sequencing, SARS$\mathrm{CoV}-2$ is a beta-coronavirus closely related to SARS virus.

This article is part of the Topical Collection on Covid-19

Kamla Al-Wahaibi

dr.alwahaibi@gmail.com

1 National Heart Center, Royal Hospital, P.O. Box 1331, Postal Code 111 Muscat, Oman

2 Division of Cardiology, Department of Medicine, Armed Forces Hospital, P.O. Box 726, Muscat Postal Code 111, Oman
The rapid spread of this diseases has quickly progressed to a global health concern. As of August 1st, 2020 more than 17.5 million people have been infected worldwide [1]. Commonly reported presenting symptoms of COVID-19 are fever, cough and shortness of breath. Less frequently reported symptoms are muscular aches, anorexia, malaise, sore throat, nasal congestion, dyspnea, and headache [2]. Lungs appear to be the primary organ involved in COVID 19 with a range of disease severity including asymptomatic subclinical infection, severe pneumonia progressing and acute respiratory distress syndrome [3]. Fortunately, the majority of patients with COVID-19 develop mild or uncomplicated illness; however, around 14\% develop serious illness that requires hospitalization and oxygen supplementation and 5\% require admission to critical care facilities [4]. Complications of severe COVID-19 infection include acute respiratory disease syndrome (ARDS), septic shock and multi- 
organ failure which may characterize by acute kidney and cardiac injury [4]. Currently, diagnosis is through SARS-CoV-2 real-time reverse transcriptase polymerase chain reaction diagnostic panel using upper and lower respiratory specimens [5].

Cardiac injury is a common condition among COVID-19 hospitalized patients [6] and is associated with an increased risk of in-hospital mortality [7]. A recently published systemic review [8] looked at more than 10 studies from Italy, USA, and China, has highlighted that myocardial injury is not uncommon in setting of COVID-19, and can lead to higher mortality in hospitalized patients. Cappannoli et al. [9] has described in a review that how patients with COVID-19 may share many characteristics with patients who have CVDs, making it often difficult to differentiate some clinical manifestations while explain why this infection is more severe in patients with underlying cardiovascular risk factors. The exact pathophysiological mechanisms that lead to myocardial injury caused by COVID19 are not well understood [6]. The postulated mechanisms include direct damage to the cardiomyocytes, systemic inflammation, myocardial interstitial fibrosis, interferon mediated immune response and exaggerated cytokine response by Type 1 and 2 helper T cells, in addition to coronary plaque destabilization, and hypoxia [6, 7]. Cardiac Injury may manifest as severe myocarditis with reduced systolic function, and elevated troponin hs-TNI $[10,11]$. Patients with pre-existing cardiovascular diseases are more prone to SARS-CoV-2 infection and they are more likely to develop stormy course [12]. Moreover, cardiovascular related comorbidities were attributed to severe COVID-19; these are arrhythmia, hypertension, cardiomyopathy and coronary heart disease $[6,12]$. Furthermore, there is a growing evidence in recently published studies to support that patients developing myocardial injury from SARS-CoV-2 are at a higher risk of in-hospital mortality [13]. Shi et al. [14] reported that among 416 patients with confirmed COVID-19, $19.7 \%$ developed cardiac injury during hospitalization; notably, mortality rate was higher in patients with cardiac injury compared with those without.

Cardiovascular diseases (CVDs) accounted for huge burden to healthcare systems worldwide [15]. In Oman, CVDs are the leading cause of death and were linked to significant morbidity [16]. In a report from the ministry of health in 2013, $30 \%$ of deaths reported were related to CVDs [16]. In this study we aim to describe characteristics of patients with COVID-19 and cardiac injury, and to examine the potential higher mortality in this high risk population.

\section{Methods}

This retrospective study was conducted with approval from research ethical and administrative committees' at participating centers. Data was obtained from subjects records preauthorized to be accessed for research purposes. Approvals were obtained from the following bodies; The Scientific Research Committee at the Royal Hospital, Muscat, Oman (Approval No. SRC\#46/2020 dated May 10th, 2020) and Forces Medical Services Medical Ethics Committee Armed Forces Hospital, Muscat, Oman (Approval No. FMC-MEC 001/2020, dated May 4th, 2020 citing committee meeting on April 30th, 2020). We included Patients, above the age of 14 years, hospitalized with laboratory-confirmed COVID-19 between March 11th, 2020, and June 27th, 2020, to any of two tertiary care centers at Muscat, the capital major metropolitan area in Oman with the highest prevalence of COVID-19 in the country. One of participating center was assigned to receive patients with severe COVID-19 and the other received unclassified cases. World Health Organization interim guidance was used to diagnose all enrolled patients in our cohort. A total of 143 patients were identified and all were included. Electronic medical records were reviewed for demographic characteristics, clinical data (symptoms, comorbidities, laboratory findings, treatments, complications, and outcomes), and laboratory tests. Cardiac injury defined as blood levels of cardiac biomarkers (hs-TNI) above the 99thpercentile upper reference limit, regardless of new abnormalities in electrocardiography and echocardiography. Data was analyzed using JMP Pro 14 and SAS software (2018 SAS Institute Inc., Cary, NC) and presented. $P$ value of $<0.050$ was considered statistically significant.

\section{Results}

\section{Demographic and Basic Characteristics}

Total of 143 patients hospitalized with confirmed COVID-19 to participating centers during the study period between March 11, 2020, and June 27, 2020 were included. Of them, 49 (34.3\%) had underlying CVD including hypertension, coronary heart disease, rhythm disturbance or cardiomyopathy and 31 patients $(21.7 \%)$ had cardiac injury as indicated by elevated hs-TNI levels and 112 patients $(78.3 \%)$ had no elevation in hs-TNI recorded. Age of study population was $49.36 \pm 15.32$ years and $124(86.7 \%)$ were male. Fever was the most common reported symptom (129 patients [84\%]). Cough, shortness of breath, diarrhea, chest pain, and sore throat were presenting symptoms for 99 patients (69\%); 76 patients (53\%), 24 patients (17\%), 21 patients (15\%), and 15 patients $(10 \%)$ respectively. Less commonly reported symptoms included: Nausea and/or vomiting (7 patients [5\%]), headache (6 patients [4\%]), rhinorrhea (6 patients [4\%]), dysuria (2 patients [1\%]), and altered mental status (1 patients $[0.6 \%])$.

Common co-existing conditions included diabetes mellitus (52 patients [36.4\%]) and hypertension (46 patients [32.2\%]). Obesity, which is defined as body mass index (BMI) $>30$, 
was noted in 23 patients (16\%). Of these 143 patients, 6 $(4.2 \%)$ and $3(2.1 \%)$ had coronary heart disease and cerebrovascular disease, respectively. The proportion of chronic heart failure, chronic renal failure, chronic obstructive pulmonary disease, smoking and cancer, was $4.2 \%$ (5 patients), $8.4 \%$ (12 patients), $7 \%$ (10 patients), $4.9 \%$ (7 patients), and 2\% (3 patients), respectively. Table 1 summarizes these findings.

Compared with patients without myocardial injury, patients with cardiac injury were older (median [range] age, 61 [33-89] years vs 44 [22-94] years; $P<0.0001$ ). Fever, followed by cough, was the leading symptom in the both cohorts. Interestingly, chest pain as a presenting symptom was higher although not statistically significant comparing non-cardiac injury group to the cardiac injury group $(15.3 \%$ vs $12.9 \%$, P 0.749 ). Moreover, patients who developed cardiac injury compared with those without cardiac injury had

Table 1 Baseline characteristics $(n=143)$

\begin{tabular}{|c|c|}
\hline Age (years) & $49.36 \pm 15.32$ \\
\hline \multicolumn{2}{|l|}{ Gender $(n) \%$} \\
\hline Male & (124) $86.7 \%$ \\
\hline Female & [19] $13.3 \%$ \\
\hline \multicolumn{2}{|l|}{ Presenting symptom(s), \% } \\
\hline Fever & $84 \%$ \\
\hline Cough & $69 \%$ \\
\hline Breathlessness & $53 \%$ \\
\hline Diarrhea & $17 \%$ \\
\hline Chest pain & $15 \%$ \\
\hline Sore throat & $10 \%$ \\
\hline Loss of appetite & $8 \%$ \\
\hline Muscle ache & $8 \%$ \\
\hline Fatigability & $8 \%$ \\
\hline Nausea and/or vomiting & $5 \%$ \\
\hline Headache & $4 \%$ \\
\hline Rhinorrhea & $4 \%$ \\
\hline Dysuria & $1 \%$ \\
\hline Altered mental status & $0.6 \%$ \\
\hline \multicolumn{2}{|l|}{ Comorbidities/risk factors, $\%$} \\
\hline Hypertension & $32.2 \%$ \\
\hline Diabetes mellitus & $36.4 \%$ \\
\hline Dyslipidemia & $7.7 \%$ \\
\hline Coronary heart disease & $4.2 \%$ \\
\hline Cerebrovascular disease & $2.1 \%$ \\
\hline Chronic heart failure & $3.5 \%$ \\
\hline Chronic kidney disease & $8.4 \%$ \\
\hline Smoking & $4.9 \%$ \\
\hline Cancer & $2 \%$ \\
\hline $\mathrm{BMI}>30$ & $16 \%$ \\
\hline Average hospitalization (days) & $11.8 \pm 8.7$ \\
\hline Average duration of symptoms (days) & $5.1 \pm 3.3$ \\
\hline
\end{tabular}

significantly more comorbidities (Table 2 ) including: hypertension (19 [61.3\%] vs 27 [24.1\%], $P<0.0001)$, diabetes (17 [54.8\%] vs 35 [31.3\%], P 0.0155), dyslipidemia (6 [19.4\%] vs $5[4.5 \%], P 0.0059)$ cerebrovascular disease $(3[9.7 \%]$ vs 0 $[0 \%], P<0.001)$, chronic heart failure $(5[16.1 \%]$ vs $0[0 \%]$, $P<0.00001)$, chronic obstructive pulmonary disease $(6$ [19.4\%] vs 4 [3.6\%], $P$ 0.002). However, there was no difference in other comorbidities including chronic kidney disease (5 [16.1\%] vs 7 [6.2\%], P 0.078), coronary heart disease (2 [6.5\%] vs 4 [3.6\%], $P$ 0.477), and obesity (7 [22.6\%] vs 16 $[14.3 \%], P 0.267)$. Cancer of any type was found in less than $4 \%$ of both cohorts with no statistical significant difference.

\section{Treatment, Complications, and Clinical Outcomes}

Patients with cardiac injury had presented to hospital in shorter period from the onset of their symptoms compared with patients who did not develop cardiac injury $(4.5 \pm 3.4$ vs $5.3 \pm 3.2$ days, $P$ 0.0216). The majority of our cohort required invasive mechanical ventilation on admission (67 patients, $46.9 \%$ ). Around quarter of patients in the study did not required oxygen support on admission; however, had other reason for admission.

The vast majority of study population received antibiotic (antibacterial) (139 [97.2\%]). Other therapeutic interventions included antivirals (96 [68.5\%]), glucocorticoids (55 [38.5\%]), and convalescent plasma infusion (63 [44\%]). Anti-interleukin drugs such as tocilizumab and anakinra were used in $48(33.5 \%)$ and $3(2 \%)$ patients respectively. Plasma exchange was used in 27 patients [18.8\%]). Table 3 summarizes treatments and interventions.

Notably, 19 patients (13.2\%) from study population required renal replacement therapy. Overall, 75 patients (52.5\%) had ARDS, and 34 patients (23.8\%) had acute kidney injury during hospitalization. Other complications included electrolyte disturbance (11 [7.7\%]), and anemia (16 [11.2\%]). On follow-up, 24 patients (16.8\%) died, 108 patients $(75.5 \%)$ were discharged, and the rest (10 [7.0\%]) remained hospitalized by the end of the study period. Compared with those without cardiac injury, patients with cardiac injury required more invasive mechanical ventilation (24 [77.4\%] vs 43 [38.4\%]; $P$ 0.00012). The use of antibiotics (antibacterial) treatment (31 [100\%] vs 108 [96.4\%]), glucocorticoids (23 [74.2\%] vs 32 [28.3\%]), and antivirals (23 [74.2\%] vs 75 [67.0\%]) was also higher in patients with cardiac injury than in those without cardiac injury. Complications were more prevalent among patients with cardiac injury than those without cardiac injury; these included ARDS (27 [87\%] vs 48 [42.9\%]; $P<0.00001$ ), acute kidney injury (21 [67.7\%] vs $13[11.6 \%] ; P<0.00001)$, drop in hemoglobin, or anemia (12 [38.7\%] vs 4 [3.6\%]; $P<0.00001)$. Figure 1 illustrates these findings. 
Table 2 Cardiac injury and COVID-19 $(n=143)$

\begin{tabular}{|c|c|c|c|}
\hline Variable & Cardiac $(n=31)$ & $\begin{array}{l}\text { Non-cardiac } \\
(n=112)\end{array}$ & $P$ value \\
\hline Age (years) & $59.03 \pm 14.83$ & $46.68 \pm 14.40$ & $<0.0001$ \\
\hline Duration of symptoms prior to hospitalization (days) & $4.5 \pm 3.4$ & $5.3 \pm 3.2$ & 0.0216 \\
\hline \multicolumn{4}{|l|}{ Leading symptoms, $\%$} \\
\hline Fever & $87 \%$ & $83 \%$ & 0.589 \\
\hline Chest pain & $12.9 \%$ & $15.3 \%$ & 0.749 \\
\hline Cough & $67.7 \%$ & $69.6 \%$ & 0.841 \\
\hline Breathlessness & $45.1 \%$ & $55.3 \%$ & 0.312 \\
\hline Diarrhea & $16.1 \%$ & $16.9 \%$ & 0.912 \\
\hline \multicolumn{4}{|l|}{ Comorbidities/risk factors } \\
\hline Hypertension & $61.3 \%$ & $24.1 \%$ & $<0.0001$ \\
\hline Diabetes & $54.8 \%$ & $31.3 \%$ & 0.0155 \\
\hline Coronary heart disease & $6.5 \%$ & $3.6 \%$ & 0.477 \\
\hline Dyslipidemia & $19.4 \%$ & $4.5 \%$ & 0.0059 \\
\hline Cerebrovascular disease & $9.7 \%$ & $0 \%$ & $<0.001$ \\
\hline Smoking & $12.9 \%$ & $2.7 \%$ & 0.019 \\
\hline Chronic heart failure & $16.1 \%$ & $0 \%$ & $<0.00001$ \\
\hline Cancer & $3.2 \%$ & $1.8 \%$ & 0.617 \\
\hline Chronic kidney disease & $16.1 \%$ & $6.2 \%$ & 0.078 \\
\hline COPD/asthma & 19.4 & $3.6 \%$ & 0.002 \\
\hline $\mathrm{BMI}>30$ & $22.6 \%$ & $14.3 \%$ & 0.267 \\
\hline \multicolumn{4}{|l|}{ Interventions, $\%$} \\
\hline Requiring ventilation support on admission & $77.4 \%$ & $38.4 \%$ & 0.00012 \\
\hline Duration on invasive ventilation (days) & $14.3 \pm 9.5$ & $10.0 \pm 10.1$ & 0.531 \\
\hline Renal replacement therapy & $48.4 \%$ & $3.6 \%$ & $<0.00001$ \\
\hline \multicolumn{4}{|l|}{ Hospitalization outcome by end of study duration, $\%$} \\
\hline Remained hospitalized & $13.4 \%$ & $5.4 \%$ & 0.144 \\
\hline Discharged & $33.3 \%$ & $87.5 \%$ & $<0.00001$ \\
\hline Death & $53.3 \%$ & $7.1 \%$ & $<0.00001$ \\
\hline
\end{tabular}

\section{Cardiac Events}

We looked into cardiac events during the course of hospitalization of the study population as well as comparing these events in those with cardiac injury and those without, including arrhythmia and ST-elevation myocardial infarction. Tachy-arrhythmias were the most common rhythm disturbance reported. Atrial tachyarrhythmia was seen in $4(12.9 \%)$ patients with cardiac injury while only seen in $1(0.9 \%)$ patient without documented cardiac injury. Ventricular arrhythmia developed in $1(3.2 \%)$ patient in the cardiac injury group vs. 2 (1.8) patients in non-cardiac group. Bradyarrhythmia occurred in $3(9.7 \%)$ in cardiac injury group compared with $6(5.4 \%)$ in non-cardiac injury group. Out of patients who had cardiac injury, 2 patients $(6.5 \%)$ presented with ST-elevation myocardial infraction.

\section{Cardiac Injury and Mortality}

The mortality rate was remarkably higher in patients with cardiac injury compared with those without cardiac injury $(16[53.3 \%]$ vs 8 [7.1\%]; $P<0.00001)$.

\section{Mortality of Patients with COVID-19 With/Without CVD and With/Without Elevated hs-TNI Levels}

Further analysis of mortality in this retrospective cohort revealed that, out of 143 patients included in this study, 3.62\% ( 3 of 83 ) with normal hs-TNI levels without underlying CVD, $17.24 \%$ (5 of 29) with normal hs-TNI levels with underlying CVD, $63.6 \%$ (7 of 11) with elevated hs-TNI levels without underlying CVD and $45 \%$ (9 of 20) with elevated hs-TNI levels with underlying CVD died during hospitalization. Figure 2 illustrates these findings. 
Table 3 Treatment and interventions

\begin{tabular}{ll} 
Initial oxygen treatment, $\%$ & \\
Oxygen supplementation via mask & $25.9 \%$ \\
Non-invasive ventilation & $2.8 \%$ \\
Invasive ventilation & $46.9 \%$ \\
No oxygen needed & $24.4 \%$ \\
Duration on invasive ventilation (days) $(n=63)$ & $11.5 \pm 10.0$ \\
Therapeutic interventions, $\%$ & \\
Antivirals & $68.5 \%$ \\
Antibiotics & $97.2 \%$ \\
Tocilizumab & $33.5 \%$ \\
Convalescent plasma & $44 \%$ \\
Anakinra & $2 \%$ \\
Plasma exchange & $18.8 \%$ \\
Renal replacement therapy & $13.2 \%$ \\
\hline
\end{tabular}

Noteworthy, patients who have died in the elevated hs-TNI levels without underlying CVD group were younger compared with patients with elevated hs-TNI levels and underlying CVD; (age median 55.4 (33-76) vs 70.0 (53-80). Male gender was the predominant in the two groups with 1 female in each group. Interestingly, deceased patients in the elevated hs-TNI levels without underlying CVD group had higher Ddimer and LDH levels than deceased patients in the elevated hs-TNI level with underlying CVD group.

\section{Discussion}

This study describes the association between myocardial injury, underlying CVD and mortality and unfavorable outcomes in patients with COVID-19.

Our study showed apparent association between cardiac injury and mortality in patients with COVID-19. Among 143 patients with COVID-19, 31 (21.7\%) who had significantly higher mortality. In cardiac injury group, the median duration from illness onset to death was 17.8 [1-15] days; a figure that is comparable to published data in literature. Shi et al. [14] described in a retrospective cohort of patients with COVID-19 that $19.7 \%$ had cardiac injury with mortality rate of $51.2 \%$ compared with $4.5 \%$ in non-cardiac injury group. Notably, there was a clear trend towards increase mortality in patients with underlying CVD as evident by almost 5 folds increase in mortality between the group with normal troponin with or without CVD (3.6\% vs $17.2 \%)$. It is also noteworthy that $82.8 \%$ of patients with underlying CVD but normal hs-TNI level had relatively better outcome compared with patients with elevated hs-TNI level but without underlying CVD (mortality: $17.2 \%$ vs $63.6 \%$ ). These results suggest that myocardial biomarkers should be utilized for risk stratification in CVD patients who develop COVID-19 infection. In a retrospective study and similar to our findings, Guo et al. [17] reported that highest mortality and the shortest survival term was seen in patients with underlying CVD (defined as patients with cardiomyopathy, coronary artery disease, or hypertension) and elevated hs-TNI level. The exact reasons why patients with underlying CVD are more vulnerable to more severe COVID-19 disease remain unclear. However, few theories have been postulated including direct viral invasion, systemic inflammatory response, destabilized coronary plaque and hypoxemia [6].

Chronic cardiovascular disease, particularly coronary artery disease and heart failure, can worsen in viral infection setting as a result of the mismatch between higher metabolic demand caused by infection and decreased cardiac reserve; subsequently, these mechanisms, may lead to ischemia [18]. In addition to these and in relation to severe systemic inflammatory response, inflammatory activity within the coronary atherosclerotic plaques is intensified, rendering them vulnerable to rupture [19]. A recently published epidemiological study [20] has demonstrated that plasma levels of cytokines including interleukin (IL)-2, IL-7, IL-10, granulocyte-colony

Fig. 1 Complications

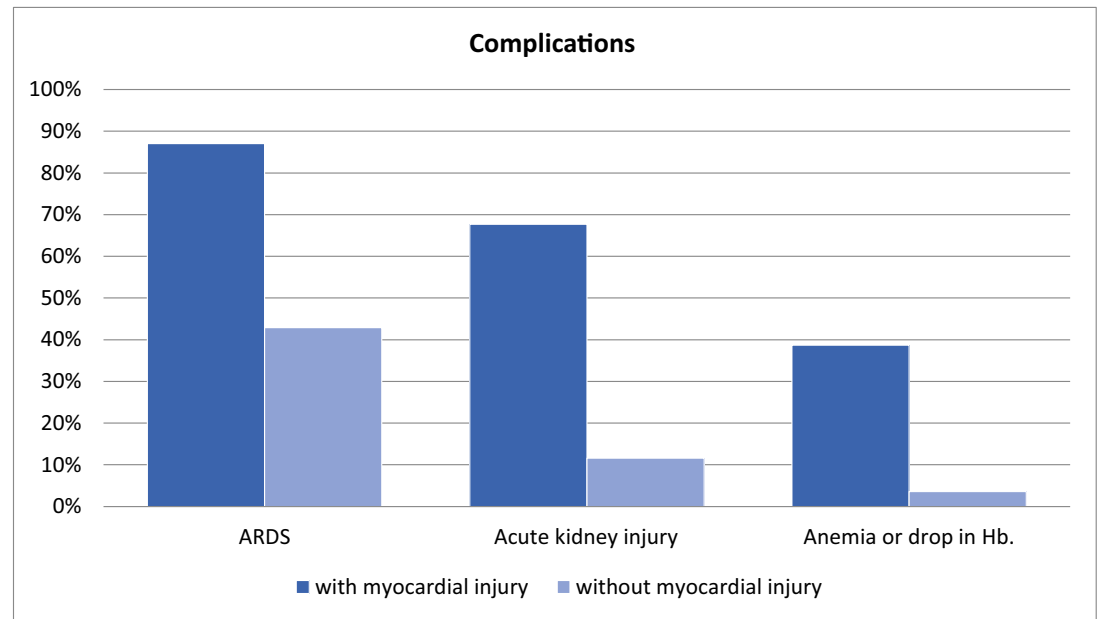


Fig. 2 Mortality of patients with coronavirus disease 2019 (COVID-19) with and without cardiovascular disease (CVD) in comparison to with and without elevated troponin (hs-TNI)
Mortality of patients with Coronavirus Disease 2019 (COVID-

19) with and without Cardiovascular Disease (CVD) in comparison to with and without Elevated Troponin (hs-TNI)

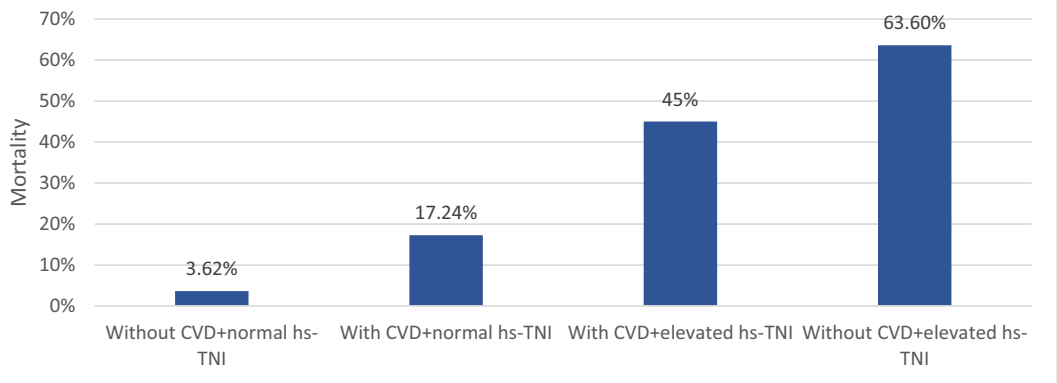

stimulating factor, IgG-induced protein 10, macrophage inflammatory protein 1 -alpha and tumor necrosis factor $\alpha$ were all elevated, above reference range, in patients with COVID19 who were admitted to the intensive care unit. This exaggerated cytokine response by type 1 and type 2 helper T cells resulting in a cytokine storm can contribute to cardiac injury [20].

Our study did also show that there may be other prognostic factors that can predict poor outcome which include D-dimer and LDH levels on admission. D-dimer, a product of lysis of cross-linked fibrin, is an important marker of thrombosis or coagulation activation [21]. Average D-dimer level on admission in those who died in the elevated hs-TNI level without underlying CVD group (mortality; 63.6\%) was around four folds (13.3 vs $3.3 \mu \mathrm{g} / \mathrm{mL}$ ) the average in the elevated hs-TNI level with underlying CVD (mortality; 45\%). A retrospective cohort study has found that among 343 patients with COVID19 , mortality in patients with D- dimer levels $\geq 2.0 \mu \mathrm{g} / \mathrm{mL}$ was higher than that in patients with D-dimer levels $<2.0 \mu \mathrm{g} / \mathrm{mL}$ $(P<.001)$ [22]. LDH could be another important prognostic marker. The mean LDH in our study in patients with elevated hs-TNI level without underlying CVD was numerically higher at 1366 compared with 659 in patients with elevated hs-TNI level but without underlying CVD. LDH is essential for glucose metabolism, particularly in conversion of pyruvate to lactate. LDH exists in all human cells, particularly in cardiac and liver cells, and Its secretion is triggered by cell membrane necrosis $[23,24]$. A multi-center study which included more than a thousand patients with COVID-19 patients has linked COVID-19 severity to elevated LDH levels [25]. LDH level also correlated to CT scans findings of severe pneumonia [26].

Patients with cardiac injury were more prone for complications such as (ARDS, $87 \%$ vs $42.9 \%, P<0.00001$, acute kidney injury, $67.7 \%$ vs $11.6 \%, \%, P<0.00001$, drop in hemoglobin or Anemia, $38.7 \%$ vs $3.6 \%, P<0.00001) .48 .4 \%$ of cardiac injury patients required renal replacement therapy compared with $3.6 \%$ in non-cardiac injury group $(P<0.00001)$. Our figures are significantly higher than these reported from similar studies. For instance, Shi et al. [14] found in their study that $58.5 \%$ of cardiac injury patients developed ARDS, $8.5 \%$ had AKI, while anemia developed in $4.9 \%$ of the cardiac injury patients. While our report of tendency to unfavorable clinical course and outcome remained consistent with findings in literature, significantly worse outcome can be explained by one of the limitation of our study as one of the designated study sites was required to receive patient with severe COVID 19 mostly.

Since patients with underlying CVD are more likely to experience adverse events in setting of myocardial injury and COVID-19 including higher risk of death, it is essential to triage patients with COVID-19 according to the presence of underlying CVD and evidence of myocardial injury for prioritized treatment and possible special treatment strategies consideration.

\section{Limitations}

Relatively small number of enrolled patients can affect statistical reflections. However, in view of the nature of emerging pandemic like COVID-19 and the fact that patients with CVD are among the highest risk and utilization of healthcare systems globally, it was still feasible to report our findings at this time. Larger cohort study is needed to verify our conclusions. One of the designated study sites received severe cases; however, we think that we were able to clearly find that myocardial injury remains an independent risk factor in setting of COVID-19 and our findings are consistent with the growing evidence.

\section{Conclusions}

Myocardial injury is not uncommon among patients admitted with COVID 19 infection and has significant association to higher mortality and poor outcomes. Further studies and meta- 
analysis are needed to replicate this finding and characterize further other predictive variables.

\section{Compliance with Ethical Standards}

Conflict of Interest The authors declare that they have no conflict of interest.

\section{References}

1. COVID-19 Map - Johns Hopkins Coronavirus Resource Center. Accessed August 1, 2020. https://coronavirus.jhu.edu/map.html

2. Stokes EK, Zambrano LD, Anderson KN, et al. Coronavirus Disease 2019 Case Surveillance - United States, January 22-May 30, 2020. MMWR Morb Mortal Wkly Rep. 2020;69(24):759-65. https://doi.org/10.15585/mmwr.mm6924e2.

3. Yuki K, Fujiogi M, Koutsogiannaki S. COVID-19 pathophysiology: a review. Clin Immunol. 2020;215:108427. https://doi.org/10. 1016/j.clim.2020.108427.

4. Clinical management of COVID-19. Accessed August 1, 2020. https://www.who.int/publications/i/item/clinical-management-ofcovid-19

5. Fang FC, Naccache SN, Greninger AL. The laboratory diagnosis of COVID-19-frequently-asked questions. Clin Infect Dis Published online June. 2020;8. https://doi.org/10.1093/cid/ciaa742.

6. Babapoor-Farrokhran S, Gill D, Walker J, Rasekhi RT, Bozorgnia B, Amanullah A. Myocardial injury and COVID-19: possible mechanisms. Life Sci. 2020;253:117723. https://doi.org/10.1016/ j.lfs. 2020.117723 .

7. Li X, Pan X, Li Y, An N, Xing Y, Yang F, et al. Cardiac injury associated with severe disease or ICU admission and death in hospitalized patients with COVID-19: a meta-analysis and systematic review. Crit Care. 2020;24(1):468. https://doi.org/10.1186/s13054020-03183-z.

8. Zou F, Qian Z, Wang Y, Zhao Y, Bai J. Cardiac injury and COVID19: a systematic review and meta-analysis. CJC Open Published online June. 2020;23:386-94. https://doi.org/10.1016/j.cjco.2020. 06.010 .

9. Cappannoli L, Scacciavillani R, Iannaccone G, Anastasia G, di Giusto F, Loria V, et al. 2019 novel-coronavirus: cardiovascular insights about risk factors, myocardial injury, therapy and clinical implications. Chronic Dis Transl Med Published online June. 2020;13. https://doi.org/10.1016/j.cdtm.2020.06.002.

10. Weckbach LT, Kellnar A, Stremmel C, Stark K, Kääb S. COVID19: a cardiological point-of-view. Dtsch Med Wochenschr. 2020;145(15):1063-7. https://doi.org/10.1055/a-1164-4161.

11. Ho JS, Sia C-H, Chan MY, Lin W, Wong RC. Coronavirusinduced myocarditis: a meta-summary of cases. Heart Lung. 2020;49(6):681-5. https://doi.org/10.1016/j.hrtlng.2020.08.013.

12. Yang J, Zheng Y, Gou X, Pu K, Chen Z, Guo Q, et al. Prevalence of comorbidities and its effects in patients infected with SARS-CoV-2: a systematic review and meta-analysis. Int J Infect Dis. 2020;94: 91-5. https://doi.org/10.1016/j.ijid.2020.03.017.

13. He XW, Lai JS, Cheng J, et al. Impact of complicated myocardial injury on the clinical outcome of severe or critically ill COVID-19 patients. Zhonghua Xin Xue Guan Bing Za Zhi. 2020;48(0):E011. https://doi.org/10.3760/cma.j.cn112148-20200228-00137.

14. Shi S, Qin M, Shen B, Cai Y, Liu T, Yang F, et al. Association of cardiac injury with mortality in hospitalized patients with COVID19 in Wuhan, China. JAMA Cardiol. 2020;5(7):802-10. https://doi. org/10.1001/jamacardio.2020.0950.

15. Roth GA, Johnson C, Abajobir A, Abd-Allah F, Abera SF, Abyu G, et al. Global, regional, and national burden of cardiovascular diseases for 10 causes, 1990 to 2015. J Am Coll Cardiol. 2017;70(1): 1-25. https://doi.org/10.1016/j.jacc.2017.04.052.

16. Al-Mawali A. Non-communicable diseases: shining a light on cardiovascular disease, Oman's biggest killer. Oman Med J. 2015;30(4):227-8. https://doi.org/10.5001/omj.2015.47.

17. Guo T, Fan Y, Chen M, Wu X, Zhang L, He T, et al. Cardiovascular implications of fatal outcomes of patients with coronavirus disease 2019 (COVID-19). JAMA Cardiol. 2020;5(7):1-8. https://doi.org/ 10.1001/jamacardio.2020.1017.

18. Xiong T-Y, Redwood S, Prendergast B, Chen M. Coronaviruses and the cardiovascular system: acute and long-term implications. Eur Heart J. 2020;41(19):1798-800. https://doi.org/10.1093/ eurheartj/ehaa231.

19. Madjid M, Vela D, Khalili-Tabrizi H, Casscells SW, Litovsky S. Systemic infections cause exaggerated local inflammation in atherosclerotic coronary arteries: clues to the triggering effect of acute infections on acute coronary syndromes. Tex Heart Inst J. 2007;34(1):11-8.

20. Huang C, Wang Y, Li X, Ren L, Zhao J, Hu Y, et al. Clinical features of patients infected with 2019 novel coronavirus in Wuhan, China. Lancet. 2020;395(10223):497-506. https://doi. org/10.1016/S0140-6736(20)30183-5.

21. Adam SS, Key NS, Greenberg CS. D-dimer antigen: current concepts and future prospects. Blood. 2009;113(13):2878-87. https:// doi.org/10.1182/blood-2008-06-165845.

22. Zhang L, Yan X, Fan Q, Liu H, Liu X, Liu Z, et al. D-dimer levels on admission to predict in-hospital mortality in patients with Covid19. J Thromb Haemost. 2020;18(6):1324-9. https://doi.org/10. 1111/jth.14859.

23. Valvona CJ, Fillmore HL, Nunn PB, Pilkington GJ. The regulation and function of lactate dehydrogenase a: therapeutic potential in brain tumor. Brain Pathol. 2016;26(1):3-17. https://doi.org/10. 1111/bpa.12299.

24. Han Y, Zhang H, Mu S, et al. Lactate dehydrogenase, an independent risk factor of severe COVID-19 patients: a retrospective and observational study. Aging (Albany NY). 2020;12(12):11245-58. https://doi.org/10.18632/aging.103372.

25. Guan W, Ni Z, Hu Y, Liang WH, Ou CQ, He JX, et al. Clinical characteristics of coronavirus disease 2019 in China. N Engl J Med. 2020;382(18):1708-20. https://doi.org/10.1056/NEJMoa2002032.

26. Xiong Y, Sun D, Liu Y, Fan Y, Zhao L, Li X, et al. Clinical and high-resolution CT features of the COVID-19 infection: comparison of the initial and follow-up changes. Investig Radiol. 2020;55(6):332-9. https://doi.org/10.1097/RLI. 0000000000000674 .

Publisher's Note Springer Nature remains neutral with regard to jurisdictional claims in published maps and institutional affiliations. 